\title{
Serological Studies on Francisella tularensis, Francisella novicida, Yersinia philomiragia, and Brucella abortus
}

\author{
SHOICHIRO OHARA, TADASHI SATO, and MORIO HOMMA
}

\author{
The Laboratory of Ohara General Hospital, 6-11 Omachi, Fukushima 960, and Department of \\ Bacteriology Yamagata University School of Medicine, Yamagata 990, Japan
}

\begin{abstract}
A study was made to reveal serological relationships among Brucella abortus, Francisella tularensis, Francisella novicida, and Yersinia philomiragia in bacterial agglutination and double agar-gel diffusion tests. All antisera used for the experiments were prepared in rabbits. In agglutination tests, cross-reaction occurred only between $F$. tularensis and B. abortus. Acetone-precipitated material showed common antigens for three species-F. tularensis, $F$. novicida, and $Y$. philomiragia in agar-gel diffusion tests-whereas polysaccharide preparations were shown to be species-specific for each of the four organisms. $B$. abortus acetone material reacted only with $F$. tularensis antiserum in agar-gel diffusion. $F$. tularensis antiserum, after absorption by intact $B$. abortus organisms, failed to react with $B$. abortus both in agar-gel diffusion and agglutination tests. The results show that $B$. abortus has surface antigens in common only with $F$. tularensis and that $F$. tularensis, $F$. novicida, and $Y$. philomiragia are antigenically related.
\end{abstract}

It has been reported that patients with naturally acquired tularemia (4) or volunteers who received either killed or live tularensis vaccine (12) exhibit agglutinins for Brucella abortus.

Close relationship between Francisella tularensis and Francisella novicida was shown by gross appearance of cultures, morphology, pathogenicity for experimental animals $(6,9)$, reciprocal deoxyribonucleic acid hybridizations (11), and transformation studies $(13,14)$. With rabbit antisera, some cross-reaction between $F$. tularensis and $F$. novicida in complement fixation tests was demonstrated, whereas agglutination, passive cutaneous anaphylaxis, and hemagglutination tests were shown to be highly specific (9). These two organisms differ significantly from other members of the genus Pasteurella and a new genus: Francisella has been proposed for them $(3,9,10)$.

Yersinia philomiragia was isolated by Jensen et al. (5) from a moribund muskrat and from water samples collected in marshy areas at the Bear River Migratory Bird Refuge in Utah. Morphologically, the isolates resembled $F$. tularensis and $F$. novicida and, also, those species that are placed in the genus Yersinia (5). Deoxyribonucleic acid hybridization studies by Ritter and Gerloff (11) indicated that it is related rather more closely to Yersinia pestis and Yersinia pseudotuberculosis than to $F$. tularensis and $F$. novicida. Because of this, although the evidence is not conclusive, it has been placed in the genus Yersinia (5). However, antigenic relationships of $Y$. philomiragia to the other related bacteria, including $F$. tularensis and $F$. novicida, have not yet been studied.

The present report is concerned with serological relationships among $B$. abortus, $F$. tularensis, $F$. novicida, and $Y$. philomiragia in bacterial agglutination and double agar-gel diffusion tests using acetone-precipitated or polysaccharide antigens.

\section{MATERIALS AND METHODS}

Bacterial strains. $F$. tularensis, strain Ebina, was isolated from a patient with tularemia in the laboratory of Ohara General Hospital in 1950. F. novicida, strain U 112, and $Y$. philomiragia, strain 309-L, were supplied through the courtesy of C. R. Owen of Rocky Mountain Laboratory, Hamilton, Mont. $B$. abortus, strain Nagashima, was supplied from the Type Culture Collection of The Institute of Medical Science, University of Tokyo. They were all grown for $24 \mathrm{~h}$ at $37 \mathrm{C}$ in a pig-liver-hemoglobin medium which has been specially devised by us (S.O. and T.S.) for the growth of $F$. tularensis (7).

Antisera. Living organisms were washed four times in physiological saline by centrifugation. They were resuspended in saline and used for immunization. Rabbits were checked for absence of agglutinins against all four organisms prior to immunization. They 
were first injected subcutaneously with $0.05 \mathrm{mg}$ (wet weight) of organisms followed by three intravenous injections of $0.1 \mathrm{mg}$ at weekly intervals. The animals were bled 7 days after the last injection and the sera were stored at $-20 \mathrm{C}$. Sera were inactivated by heating at $56 \mathrm{C}$ for $30 \mathrm{~min}$ just before use. The sera did not form a precipitin band against growth medium in the agar-gel diffusion test.

Bacterial agglutination tests. Twofold dilutions of sera were made in saline and to each tube was added one drop of a saline suspension $(5 \mathrm{mg} / \mathrm{ml}$ ) of the organism to be tested. The mixtures were shaken and allowed to stand overnight at $37 \mathrm{C}$. Agglutinin titers were recorded as reciprocals of the highest dilution of serum that produced macrovisible agglutination.

Acetone-precipitated antigens. Organisms were washed four times in saline by centrifugation. The resulting pellets were suspended in the saline and were precipitated by an excess of acetone overnight at room temperature. The precipitates were collected by centrifugation, dried at room temperature, and ground to a fine powder by mortar and pestle. The dry powder was then suspended in distilled water, containing $0.1 \%$ sodium nitrate, at a concentration of $1 \mathrm{~g}$ (dry weight) per $50 \mathrm{ml}$, and stored at $4 \mathrm{C}$ until desired.

Polysaccharide antigens. The polysaccharides were prepared by the phenol-extraction method of Alexander (1). The dried organisms, after extraction with acetone, were extracted overnight with 10 volumes of liquefied phenol at $40 \mathrm{C}$. Five volumes of distilled water were then added, and the mixture was dialyzed against running tap water for 2 days. After centrifugation, three volumes of $95 \%$ ethyl alcohol were added to the supernatant and the mixture was allowed to stand for $1 \mathrm{~h}$ at $0 \mathrm{C}$. It was centrifuged, and the precipitate was washed once by precooled alcohol and dried in vacuo. The final polysaccharide powder was dissolved in 50 volumes of distilled water (weight per volume) containing $0.1 \%$ sodium nitrate and stored at $4 \mathrm{C}$ until desired.

Preparation of bacterial homogenates. Organisms were suspended in physiological saline $(1: 10)$ and treated in ice with an ultrasonic oscillator for $5 \mathrm{~min}$ (Tominaga, model UR-150P, $20 \mathrm{kc} / \mathrm{s}$ ).

Gel diffusion tests. Ouchterlony-type double diffusion tests were carried out in a $100-\mathrm{mm}$ petri dish containing a $5-\mathrm{ml}$ bottom layer and a $15-\mathrm{ml}$ top layer of $1 \%$ agar (Difco) in physiological saline with $0.1 \%$ sodium nitrate. Circular antigen and antibody reservoirs of $10-\mathrm{mm}$ diameter were made in the top agar layer. After filling the reservoirs, diffusion plates were incubated at $37 \mathrm{C}$ and examined daily for 2 weeks, and the photographs were made on the 7 th to 10 th day.

\section{RESULTS}

Bacterial agglutination. Typical results are shown in Table 1. Rabbit antisera prepared against $F$. novicida and $Y$. philomiragia agglutinated only homologous organisms. Crossagglutination was observed between $B$. abortus and $F$. tularensis. However, B. abortus antiserum agglutinated $F$. tularensis to low titers $(1: 10)$ only and sera from rabbits frequently failed to show heteroagglutinins.

Gel diffusion by acetone-precipitated antigens. Antigens of the test organisms were prepared either by acetone precipitation or by sonic vibration, and the antigenicities of both preparations were directly compared in double diffusion tests in agar using the respective homologous antisera. No gross antigenic differences were found between the two preparations from all of the organisms tested (Fig. 1). The acetone-precipitated antigens were then tested with homologous and heterologous antisera.

TABLE 1. Cross-agglutination tests by antisera from rabbit immunized with Brucella abortus, Francisella tularensis, Francisella novicida, and Yersinia philomiragia antigens

\begin{tabular}{l|r|r|r|r}
\hline \multirow{2}{*}{$\begin{array}{c}\text { Rabbit antisera } \\
\text { immunized } \\
\text { with }\end{array}$} & \multicolumn{4}{|c}{$\begin{array}{c}\text { Reciprocal of agglutination } \\
\text { titer against }\end{array}$} \\
\cline { 2 - 5 } & B. abortus & $\begin{array}{c}\text { F. tular- } \\
\text { ensis }\end{array}$ & $\begin{array}{c}\text { F. nov- } \\
\text { icida }\end{array}$ & $\begin{array}{c}\text { Y. philo- } \\
\text { miragia }\end{array}$ \\
\hline B. abortus & 160 & 10 & $<5$ & $<5$ \\
F. tularensis & 80 & 640 & $<5$ & $<5$ \\
F. novicida & $<5$ & $<5$ & 320 & $<5$ \\
Y. philomiragia & $<5$ & $<5$ & $<5$ & 80 \\
\hline
\end{tabular}
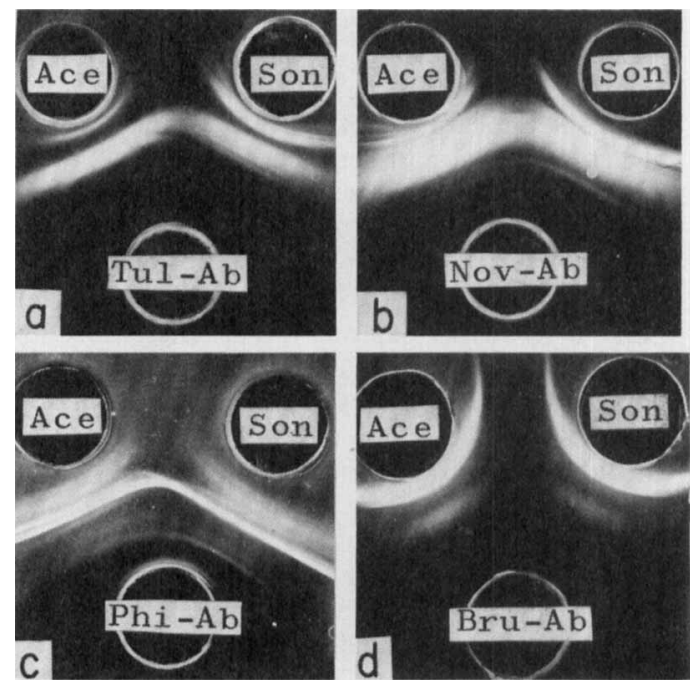

FIG. 1. Comparison of acetone-precipitated antigens with sonically disrupted antigens in gel diffusion tests. Acetone-precipitated antigens (Ace); sonicated homogenates (Son). Antigens prepared by either method were placed in the top reservoirs and homologous antisera were placed in the bottom reservoirs. (a) $F$. tularensis; (b) $F$. novicida; (c) $Y$. philomiragia; (d) B. abortus. 
$F$. tularensis antiserum exhibited many distinct precipitin lines against the acetone-precipitated antigens of all organisms (Fig. 2), disclosing common antigens between $F$. tularensis and each of the other three bacteria. Tests were then done to discover whether the antigens in the $B$. abortus preparation reacting with the antiserum against $F$. tularensis were identical with those in the $F$. novicida and $Y$. philomiragia preparations reacting with the same serum. Figure $3 \mathrm{a}$ shows that none of the precipitin lines formed between the antiserum and the $B$. abortus antigen fuse with any of the precipitin lines formed between the same serum and the other antigens. This indicates that the common antigens of $F$. tularensis and $B$. abortus are different from any of those common to $F$. tularensis, $F$. novicida and $Y$. philomiragia.

The next experiment was conducted to see the relationship between $F$. novicida antigens and $Y$. philomiragia antigens reacting with $F$. tularensis antibodies. Some, but not all, of the precipitin lines formed by the $F$. tularensis antiserum against $F$. novicida antigen apparently fuse with the lines against $Y$. philomiragia antigen (Fig. 3b). This shows not only that there exist antigens common to $F$. tularensis, and $F$. novicida, and $Y$. philomiragia, but also that some of the antigens are common to all three bacteria. The results obtained from the following experiments were consistent with the above findings (Fig. 4 and 5).

F. novicida (Fig. 4a) and $Y$. philomiragia (Fig. 4b) antisera not only reacted with homologous antigen, but also with heterologous antigens with the exception of brucella antigen.

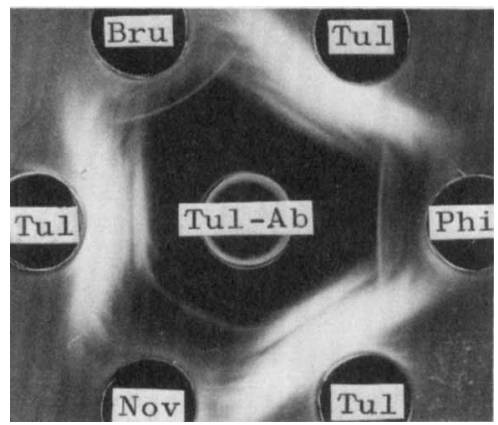

FIG. 2. Immunodiffusion analysis of tularensis antiserum by acetone-precipitated antigens from different organisms. Rabbit antiserum against $F$. tularensis was placed in the center reservoir and antigens in the peripheral reservoirs. In this and all subsequent figures, the following abbreviation was used: Bru, $B$. abortus; Tul, F. tularensis; Nov, F. novicida; Phi, $Y$. philomiragia; $A b$, antibody or antiserum.

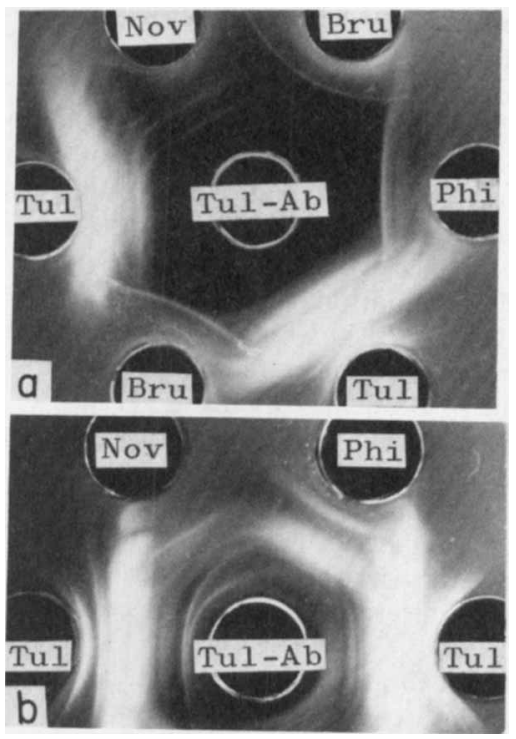

FIG. 3. Immunodiffusion analysis of acetoneprecipitated antigens of $F$. tularensis and other organisms. $F$. tularensis antiserum was placed in the center reservoir. The lines formed by brucella antigen do not fuse with any of those formed by either novicida or philomiragia antigen (a), whereas some of the lines formed by novicida antigen coalesce into those formed by philomiragia antigen (b).

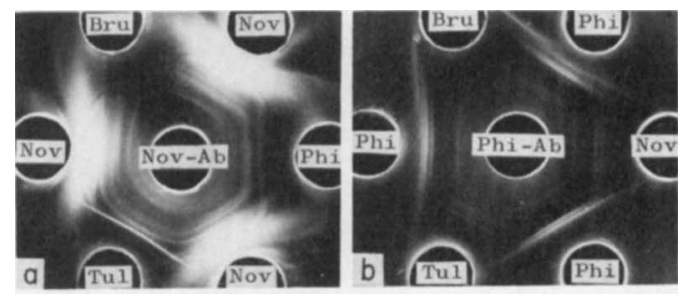

FIG. 4. Immunodiffusion analysis of novicida antiserum (a) and philomiragia antiserum (b) by acetoneprecipitated antigens from different organisms. Antisera were placed in the center reservoirs. No precipitin band is seen between either of the antisera and brucella antigen.

Novicida antiserum reveals several antigens common to tularensis and philomiragia antigens, since some of the precipitin lines formed between tularensis antigens and novicida antiserum evidently fused with the lines formed between philomiragia antigen and the same antiserum reservoir (Fig. 5a). Philomiragia antiserum also revealed antigens in common with $F$. tularensis and $F$. novicida (Fig. 5b). Overall results clearly demonstrated the existence of antigens common to $F$. tularensis, $F$. novicida, and $Y$. philomiragia. 
B. abortus antiserum, however, reacted only with homologous antigen, but not with heterologous antigens (Fig. 6); common antigens between $B$. abortus and $F$. tularensis which were readily detectable by the use of tularensis antiserum (Fig. 2 and $3 \mathrm{a}$ ) could not be detected. Perhaps the brucella antigens shared with $F$. tularensis are too weak to stimulate the antibody formation in rabbits. Heterologous antibodies in $F$. tularensis antiserum reacting with brucella antigen are specifically absorbed by intact brucella organism (Fig. 7): the resulting absorbed serum fails to agglutinate $B$. abortus although it does agglutinate $F$. tularensis (Table 2). The results suggest that a part of the heterologous antibodies in the tularensis antiserum demonstrable in agar-gel diffusion represents the heteroagglutinin to $B$. abortus.

Gel diffusion by polysaccharide antigens. The polysaccharide preparations from all of the given organisms reacted in a different way from the acetone-precipitated ones in the gel diffusion test. Antisera reacted only with the homologous polysaccharide preparations (Fig. 8a-d).

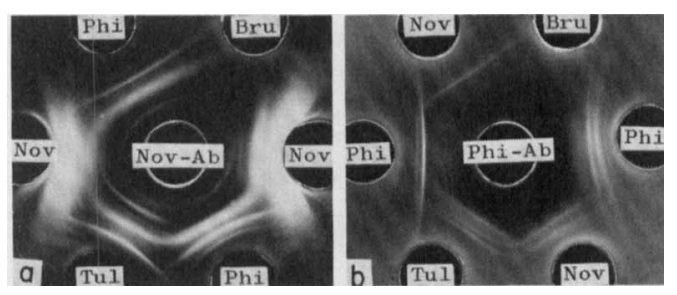

FIG. 5. Immunodiffusion analysis of acetone-precipitated antigens from different organisms, common to $F$. novicida (a) or to $Y$. philomiragia (b). Antisera were placed in the center reservoirs.

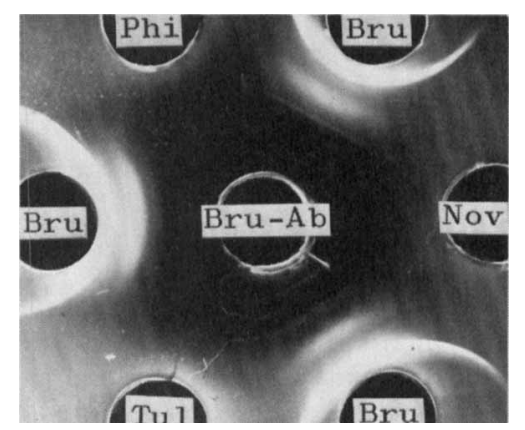

FIG. 6. Immunodiffusion analysis of brucella antiserum by acetone-precipitated antigens from different organisms. Antiserum was placed in the center reservoir.

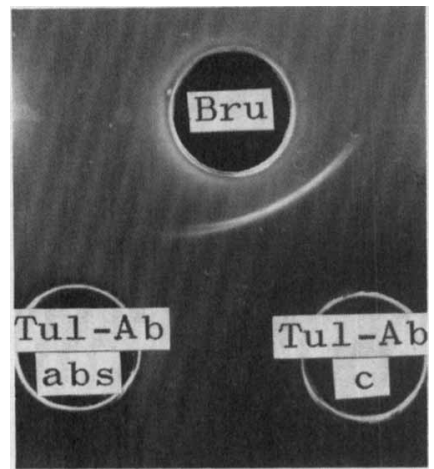

FIG. 7. Immunodiffusion analysis of tularensis antiserum after absorption by intact brucella organisms. Acetone-precipitated brucella antigen was placed in the top reservoir, absorbed serum was placed in the left bottom reservoir, and unabsorbed serum was placed in the right bottom reservoir. The absorbed serum was the same as described in Table 2. Abbreviations: abs, absorbed; c, control.

TABLE 2. Absorption of heteroagglutinin in tularensis antiserum by $B$. abortus

\begin{tabular}{l|c|c}
\hline \multirow{2}{*}{ Tularensis antiserum } & \multicolumn{2}{|c}{$\begin{array}{c}\text { Reciprocal of agglutination } \\
\text { titer against }\end{array}$} \\
\cline { 2 - 3 } & B. abortus & F. tularensis \\
\hline Before absorption & 80 & 640 \\
After absorption $^{a}$ & $<5$ & 640 \\
\hline
\end{tabular}

${ }^{a}$ Tularensis antiserum was absorbed twice with one-fifth volume of living $B$. abortus organisms followed by incubation for $5 \mathrm{~h}$ at $4 \mathrm{C}$ and centrifugation.

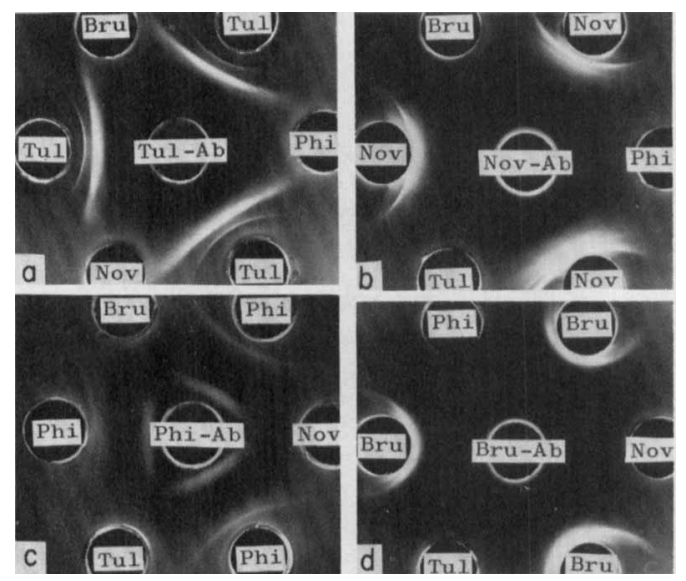

FIG. 8. Immunodiffusion analysis of polysaccha. ride preparations from different organisms. Antisera were placed in the center reservoirs and antigens in the peripheral reservoirs. Antisera: $a, F$. tularensis; $b, F$. novicida; $c, Y$. philomiragia; $d, B$. abortus. 


\section{DISCUSSION}

The present study reveals that tularensis antiserum contains heteroagglutinins to $B$. abortus, results consistent with previous reports $(4,12)$. On the other hand, brucella antiserum exhibits only low titers of heteroagglutinin to $F$. tularensis, in agreement with the findings of Carlisle et al. (2). With the above exceptions, agglutination tests with the various organisms were highly specific (Table 1). The results also confirmed the previous work of Owen et al. (9) in which no cross-agglutination between $F$. tularensis and $F$. novicida was demonstrated.

To disclose an antigenic relationship among the given organisms, studies were made in agar-gel diffusion tests using acetone-precipitated antigens. Tularensis antiserum formed distinct precipitin lines against acetone-precipitated antigens prepared from B. abortus (Fig. 2 ). This antibody completely failed to precipitate the same antigen after absorption by intact B. abortus (Fig. 7) and also failed to agglutinate the latter organism (Table 2). The results indicate that brucella antigens which react with tularensis antibodies in both tests are localized on the surface of the organism. Brucella antisera prepared in rabbits have failed so far to react with acetone-precipitated tularensis antigens in agar-gel diffusion tests. Acetone-precipitated preparations of $F$. tularensis, $F$. novicida, and $Y$. philomiragia exhibited antigens common to all three organisms in agar-gel diffusion tests (Fig. 3b, 5a, and 5b). However, no antigenic relationship has been found between $B$. abortus and $F$. novicida or $Y$. philomiragia (Fig. $4 \mathrm{a}, 4 \mathrm{~b}$, and 6).

Antigenic specificity of polysaccharide preparations of $B$. abortus (1), $F$. tularensis, and $F$. novicida (9) has been reported. The present data support this view, showing that the precipitin pattern of each of the polysaccharide antigens was characteristic and did not react with the heterologous antisera. All of the polysaccharide preparations were found to be multivalent, the result being consistent with that reported previously (8).

Genetic similarity between $F$. tularensis and $F$. novicida has been disclosed by the DNA homology test (11) and by transformation (14). On the basis of the DNA homology test, Ritter and Gerloff stated that $Y$. philomiragia is remote from $F$. tularensis and $F$. novicida but rather close to Pasteurella pestis and P. pseudotuberculosis, for which a new genus name Yersinia had been proposed (10). Taxonomic placement of $Y$. philomiragia in the genus Yersinia has been done on the basis of the above findings (5), but the hybridization experiments between $Y$. philomiragia and the other species of Yersinia seem too inconclusive to prove a definite genetic similarity of these bacteria.

There is no argument that $F$. tularensis, $F$. novicida, and $Y$. philomiragia belong to different species, since the present study has revealed serological specificity of these bacteria in agglutination and agar-gel diffusion tests with their polysaccharide preparations. On the other hand, results have also clearly demonstrated the existence in acetone-precipitated preparations of multiple antigens common to all of these three organisms, although no efforts to establish identities of these antigens have been made. Thus, more adequate study is necessary to gain a better basis for the taxonomic placement of $Y$. philomiragia, especially in relation to the genera Francisella and Yersinia.

\section{ACKNOWLEDGMENTS}

We thank C. R. Owen of Rocky Mountain Laboratory, Hamilton, Mont., for the supply of $F$. novicida, strain U 112 and $Y$. philomiragia, strain 309-L. Our thanks are also due to J. F. Bell of the same laboratory for revising the manuscript.

\section{LITERATURE CITED}

1. Alexander, M. M. 1950. A quantitative antibody response of man to infection or vaccination with Pasteurella tularensis. J. Exp. Med. 92:51-57.

2. Carlisle, H. D., V. Hinchliffe, and S. Saslaw. 1962. Immunodiffusion studies with Pasteurella tularensis antigen-rabbit antibody systems. J. Immunol. 89:638-644.

3. Dorofeev, K. A. 1947. On the classification of tularemic bacteriae. Symp. Res. Work Inst. Epidemiol. Microbiol. Chita 1:177-178.

4. Feinberg, R. J., and G. G. Wright. 1951. Factors influencing the agglutination titration in human brucellosis. J. Immunol. 67:115-122.

5. Jensen, W. I., C. R. Owen, and W. L. Jellison. 1969. Yersinia philomiragia sp. n., a new member of the Pasteurella group of bacteria, naturally pathogenic for the muskrat (Ondatra zibethica). J. Bacteriol. 100:1237-1241.

6. Larson, C. L., W. Wicht, and W. L. Jellison. 1955. A new organism resembling $P$. tularensis isolated from water. Pub. Health Rep. 70:253-258.

7. Ohara, S., and T. Sato. 1955. On cultivation of Bacterium tularense by using the pig-liver-hemoglobin medium. Fukushima Igaku Zasshi 5:533-535.

8. Ormsbee, R. A., and C. L. Larson. 1955. Studies on Bacterium tularense antigens. II. Chemical and physical characteristics of protective antigen preparations. J. Immunol. 74:359-370. 
9. Owen, C. R., E. O. Buker, W. L. Jellison, D. B. Lackman, and J. F. Bell. 1964. Comparative studies of Francisella tularensis and Francisella novicida. J. Bacteriol. 87:676-683.

10. Philip, C. B., and C. R. Owen. 1961. Comments on the nomenclature of the causative agent of tularemia. Int. Bull. Bacteriol. Nomen. Taxon. 11:67-72.

11. Ritter, D. B., and R. K. Gerloff. 1966. Deoxyribonucleic acid hybridization among some species of the genus Pasteurella. J. Bacteriol. 92:1838-1839.
12. Saslaw, S., and H. N. Carlisle. 1961. Studies with tularemia vaccines in volunteers. IV. Brucella agglutinins in vaccinated volunteers challenged with Pasteurella tularensis. Amer. J. Med. Sci. 242:166-172.

13. Tyeryar, F. J. Jr., and W. D. Lawton. 1969. Transformation of Pasteurella novicida. J. Bacteriol. 100:1112-1113.

14. Tyeryar, F. J. Jr., and W. D. Lawton. 1970. Factors affecting transformation of Pasteurella novicida. J. Bacteriol. 104:1312-1317. 